\title{
Successful treatment with pollen extract of hematospermia in patients with xanthogranolomatous prostatitis
}

\author{
Antonio Luigi Pastore ${ }^{1,2}$, Yazan Al Salhi ${ }^{1}$, Andrea Fuschi ${ }^{1}$, Alessia Martoccia ${ }^{1}$, Gennaro Velotti ${ }^{1}$, \\ Lorenzo Capone $^{1}$, Giorgio Bozzini ${ }^{3}$, Natale Porta ${ }^{4}$, Vincenzo Petrozza ${ }^{4}$, Ester Illiano ${ }^{5}$, \\ Elisabetta Costantini ${ }^{5}$, Antonio Carbone ${ }^{1,2}$ \\ ${ }^{1}$ Sapienza University of Rome, Faculty of Pharmacy and Medicine, Department of Medico-Surgical Sciences and Biotechnologies, \\ Urology Unit, Latina Italy; \\ ${ }^{2}$ Uroresearch, no profit Association for Research in Urology, Latina, Italy; \\ ${ }^{3}$ Department of Urology Mater Domini Humanitas Castellanza (VA), Italy; \\ ${ }^{4}$ Sapienza University of Rome, Faculty of Pharmacy and Medicine, Department of Medico-Surgical Sciences and Biotechnologies, \\ Pathology Unit, Latina Italy; \\ ${ }^{5}$ Department of Urology and Andrology, University of Perugia, Terni Italy.
}

\begin{abstract}
Summary Introduction: The aim of this study was to report our experience in the management of hematospermia observed in 16 patients suffering from xanthogranulomatous prostatitis.

Methods: Recurrent episodes of hematospermia were the onset symptom in all patients, and in $25 \%$ of patients it was combined with fever. All patients reported PSA value elevation and the digital rectal examination (DRE) revealed an increase of the gland size and of its consistency in all cases. In all patients, the hematospermia was treated with the oral administration of two tablets of pollen extract in a single $(1 \mathrm{~g})$ dose daily for 30 days. Results: Sixteen patients were observed between 2008 and 2016, referring hematospermia, progressive lower urinary tract symptoms (LUTS), and serum PSA level increase. To exclude the prostate cancer presence all patients were submitted to transperineal TRUS guided biopsy. In all the patients complete resolution of hematospermia was achieved treatment with pollen extract. All patients were subsequently treated for LUTS (alpha-adrenergic blockers), but none reported any significant improvement of symptoms. Basing on these pieces of evidence, after 90 days of alpha-blockers therapy, all patients underwent bipolar TURP. Histological examination of resected prostatic tissue revealed in all patients the diagnosis of xanthogranulomatous prostatitis.

Conclusions: Patients with xanthogranulomatous prostatitis especially experience irritative symptoms, sometimes combined with fever or hematospermia. Hematospermia as the onset symptom has not been reported so far. The administration of the pollen extract for 30 days was associated with a complete resolution of hematospermia.
\end{abstract}

KEY WORDS: Hematospermia; Lower urinary tract symptoms; Pollen extract; Xanthogranulomatous prostatitis.

Submitted 6 October 2018; Accepted 26 January 2019

\section{INTRODUCTION}

Granulomatous prostatitis is a non-specific inflammatory process of the prostate gland, characterized by the presence of granuloma as the main histological feature (1).

It is classified as: infectious granuloma, nonspecific gran- ulomatous prostatitis, post-biopsy granuloma, and systemic granulomatous prostatitis. Rare forms of granulomatous prostatitis include sarcoidosis and xanthogranulomatous prostatitis (2). This form is histologically similar to granulomatous prostatitis, with the prominence of foamy histiocytes, which constitute the xanthomatous component. Non-specific granulomatous prostatitis and xanthogranulomatous prostatitis are likely caused by a blockage of prostatic ducts and stasis of gland secretions. The resulting epithelial disruption leads to the escape of cellular debris, bacterial toxins, prostatic secretions, including corpora amylacea, sperm and semen into the stroma, determining an intense localized inflammatory response. The most reported onset symptom is represented by irritative lower urinary tract symptoms and a raise of serum prostate-specific antigen (PSA) (3) that mimics adenocarcinoma (4). In this study we report our experience on xanthogranulomatous prostatitis observed in 16 patients. All patients were complaining hematospermia, progressive lower urinary tract symptoms (LUTS), and increasing PSA levels. Aim of the study is to describe our successful therapeutic management of this bothering onset symptom, hematospermia, related to this rare form of prostatitis.

\section{Patients ANd methods}

All patients came to our attention complaining recurrent episodes of hematospermia (associated with fever in 25\% of patients), that represented the onset symptom of all cases. All men suffered also from irritative LUTS, mostly characterized by urinary frequency, burning, hesitancy, and nocturia.

All patients provided written informed consent. The study was conducted in accordance with the Declaration of Helsinki and was approved by the local Medical Ethical Committee (ASL LT CE approval n.08/1636/42 UROUNIV). In all patients, a PSA elevation was observed (range: 4.9$9.7 \mathrm{ng} / \mathrm{mL}$ ), with a free/total ratio always greater than $20 \%$ (range: 22-36\%). Digital rectal examination (DRE) revealed an increase in the gland volume and a consistency change 
with an irregular surface in all cases. The palpatory findings (peripheral nodule of hard consistency) and the serum total PSA level $>4 \mathrm{ng} / \mathrm{mL}$ lead us to suspect malignancy in all cases. All patients underwent an ultrasoundguided transperineal prostatic biopsy (12 cores) to exclude the presence of prostate cancer.

All patients were investigated for Chlamydia trachomatis (Ct), Ureaplasma urealyticum, Neisseria gonorrhoeae, herpes viruses (HSV 1/2) and human papillomavirus (HPV).

In all cases the hematospermia was treated with the oral administration of two tablets of DEPROX $500^{\circledR}$ in a single dose daily, in line with previous studies 5,6 and according with the manufacturer's instructions (IDI ${ }^{\circledR}$ Integratori Dietetici Italiani S.r.l, Catania, Italy). Each administration contained $1 \mathrm{~g}$ pollen extract ( $500 \mathrm{mg}$ per tablet), and vitamins B1, B2, B6, B9, B12 and PP. Statistical analysis (Student t-test for paired samples) was performed to compare the outcomes before and after medical therapy and pre- and post-operative. The statistical analysis was done using SPSS software (version 21.0; SPSS Inc., Chicago, IL). $P$ values $<0.05$ were considered as statistically significant.

\section{RESULTS}

Sixteen patients were observed between 2008 and 2016, referring hematospermia, progressive lower urinary tract symptoms (LUTS), and serum PSA level increase. In all patients the hematospermia was treated with Pollen Extract ( 2 tabs - $1 \mathrm{~g}$ daily) for 30 days with complete resolution of this symptom. The DEPROX $500^{\circledR}$ treatment was well tolerated in all the analyzed patients, and no significant drug-related side-effect was reported.

In all 16 cases the results of infection tests, and the prostate biopsy to detect cancer resulted negative. The prostatic biopsy did not allow the histological diagnosis of xanthogranulomatous prostatitis due to the poor biopsy material that did not enable the histotyping of prostatitis. Thereafter, all patients were treated for LUTS (alpha-adrenergic blockers), but none reported any significant improvement of symptoms, as revealed by IPSS questionnaire and the $\mathrm{Q}_{\max }$ registered 30 days post treatment. For this reason, all the subjects were evaluated by transrectal ultrasound examination (TRUS) and urodynamics. TRUS showed a marked inhomogeneity of prostate tissue, with several hypo-echoic and hyper-vascularized areas and calcifications along the peripheral surface of the adenoma, while pressure/flow studies showed the presence of a severe bladder outlet obstruction (BOO). Basing on these evidences, after 90 days of alpha blockers therapy, all patients underwent transurethral bipolar endoscopic resection of the prostate (TURP). Mean catheterization time was 2.8 days (range: $2-4$ days); in only one patient acute urinary retention 48 hours after catheter removal due to inflammatory condition (as revealed by DRE) occurred. PSA levels significantly decreased (below $2.0 \mathrm{ng} / \mathrm{mL}$ ) in all patients. Functional outcomes before and after alpha blockers, pre and post TURP were evaluated by IPSS, IPSS-QoL, maximum flow rate $\left(Q_{\max }\right.$ expressed in $\left.\mathrm{mL} / \mathrm{sec}\right)$, and post void residual urine volume $(\mathrm{mL})$. All patients achieved normal IPSS scores and normal uroflowmetry parameters 5 weeks after surgery. The data are summarized in Table 1.
Table 1.

Functional outcomes before and after alpha blocker's therapy, and preoperative and 3 months after TURP.

\begin{tabular}{|c|c|c|c|}
\hline & Before therapy & After therapy & $p$ value \\
\hline IPSS (SD) & $23.65(0.12)$ & $23.51(0.11)$ & 0.486 \\
\hline IPSS - QoL (SD) & $4.45(0.04)$ & $4.54(0.03)$ & 0.115 \\
\hline$Q_{\max }(S D)$ & $9.81(0.13)$ & $9.69(0.12)$ & 0.553 \\
\hline \multirow[t]{2}{*}{$\overline{P V R}(S D)$} & $112.95(0.2)$ & $113.09(0.18)$ & 0.610 \\
\hline & Preoperative & 3 months after TURP & $p$ value \\
\hline IPSS (SD) & $23.51(0.11)$ & $7.41(0.11)$ & $<0.0001$ \\
\hline IPSS QoL (SD) & $4.54(0.03)$ & $1.54(0.05)$ & $<0.0001$ \\
\hline $\mathrm{Q}_{\max }(\mathrm{SD})$ & $9.69(0.12)$ & $20.74(0.11)$ & $<0.0001$ \\
\hline PVR (SD) & $113.09(0.18)$ & $21.12(0.19)$ & $<0.0001$ \\
\hline PSA (SD) & $6.24(0.33)$ & $1.32(0.27)$ & $<0.0001$ \\
\hline \multicolumn{4}{|c|}{$\begin{array}{l}\text { SD: Standard Deviation; IPSS: International Prostate Symptom Score; } \\
\text { QoL: Quality of life; } Q_{\text {max }} \text { maximum flow rate }(\mathrm{mL} / \mathrm{sec}) ; \text { PVR; post-void residual urine } \\
\text { volume (mL); PSA; prostate specific antigen }(\mathrm{ng} / \mathrm{mL}) \text {. }\end{array}$} \\
\hline
\end{tabular}

All patients submitted to TURP reported significant outcomes at the 3 months' follow-up visit $(\mathrm{p}<0.0001)$. Histological examination of resected prostatic tissue revealed in all patients the diagnosis of xanthogranulomatous prostatitis.

\section{Discussion}

The histopathological examination of resected prostatic tissue in all our patients revealed xanthogranulomatous prostatitis with no evidence of malignancy. A non-specific granulomatous inflammation was found, the granulomas were composed of multinucleated giant cells and "xanthogranulomatous cells" (diffusely in 13 cases, focally in one patient; Figure 1). Xanthomatous histiocytes presented a small dark nuclei and abundant clear to foamy cytoplasm due to fat droplets and could be confused with prostate carcinoma. As confirmed by our case series, the final diagnosis of xanthogranulomatous prostatitis can only be achieved by histopathological examination of the prostate $(7,8)$. The histological feature of xanthogranulomatous prostatitis is the presence of macrophages with foamy cytoplasm "xanthomatous cells" (CD68+) in the mixed inflammatory infiltrate with multinucleated giant cells. Major study limitation was the small number of patients, and the absence of a placebo group of treatment. However, the low number of patients to be enrolled was not sufficient to design a placebo controlled study.

In the present study the administration of DEPROX $500^{\circledR}$ was able to achieve the complete resolution of hematospermia with a disappearance of this onset symptom in all patients within 15 days of treatment assumption, without severe side-effects. To the best of our knowledge this was the first study to evaluate this treatment for this symptom. Furthermore, hematospermia as the onset symptom has not been reported so far. Hematospermia has been sporadically reported as an accompanying symptom in very few cases (9), but only in our case series it represented the uncommon symptom of the disease onset. In $40 \%$ of all cases reporting hematospermia, an infectious condition is revealed. Other etiologic factors are inflam- 
Figure 1.

A. Low-power photomicrograph showing intense inflammatory infiltrate composed of lymphocytes, plasma cells, neutrophils and xanthogranulomatous cells in prostatic tissue (magnification 10×); B. High-power photomicrograph showing xanthomatous component of inflammatory infiltrate, composed of histiocytes with small dark nuclei and abundant clear to foamy cytoplasm (magnification 40×); C. CD68 stain showing the presence of xanthomatous histiocytes in the prostatic stroma (magnification 40×).

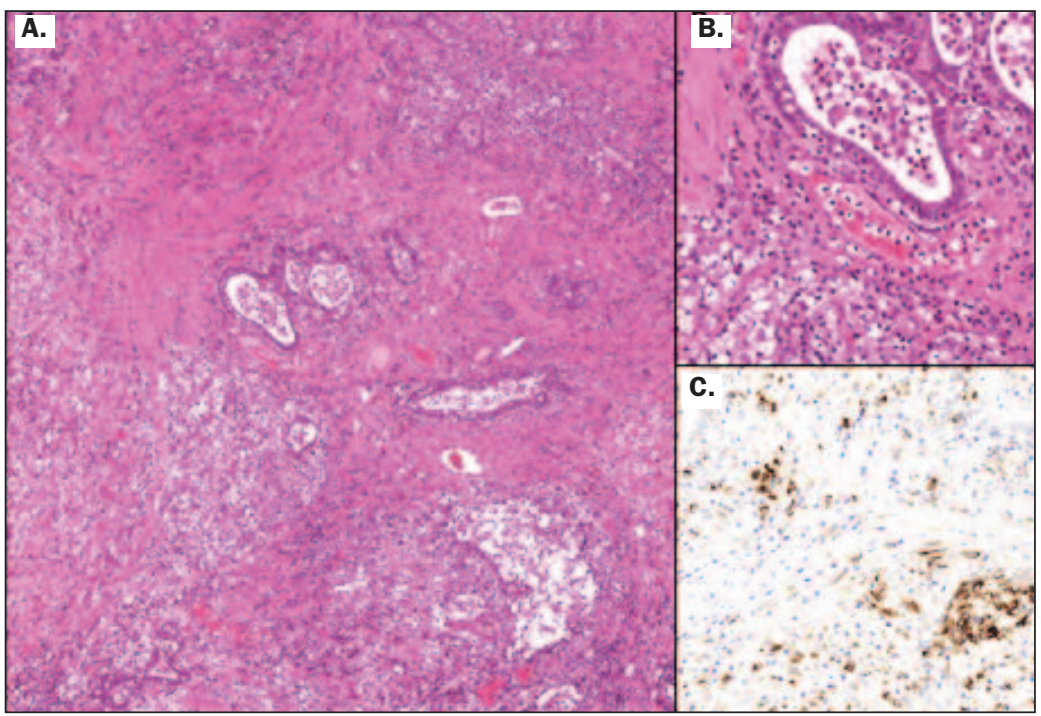

\section{REFERENCES}

1. Epstein JI, Hutchins GM. Granulomatous prostatitis: Distinction among allergic, nonspecific, and post-transurethral resection lesions. Hum Pathol. 1984; 15:818-25.

2. Shanggar K, Zulkinfli MZ, Razack AH, et al. Granulomatous prostatitis: A reminder to clinicians. Med J Malaysia. 2010; 65:21-2.

3. Speights VO, Jr, Brawn PN. Serum prostatespecific antigen levels in non-specific granulomatous prostatitis. Br J Urol. 1996; 77:408-10.

4. Srigley JR. Benign mimickers of prostatic adenocarcinoma. Mod Pathol 2004; 17:328-33

5. Cai T, Luciani LG, Caola I, et al. Effects of pollen extract in association with vitamins (DEPROX 500®) for pain relief in patients affected by chronic prostatitis/chronic pelvic pain syndrome: results from a pilot study. Urologia. 2013; 80(Suppl 22):5-10.

6. Cai T, Wagenlehner FM, Luciani LG,et al. Pollen extract in association with vitamins provides early pain relief in patients affected by chronic prostatitis/chronic pelvic pain syndrome. Exp Ther Med. 2014; 8:1032-1038.

7. Uzoh CC, Uff JS, Okeke AA. Granulomatous prostatitis. BJU Int. 2007; 99:510-2.

8. Pastore AL, Palleschi G, Fuschi A, et al. Hematospermia and xanthogranulomatous pro-

matory conditions, neoplasms and iatrogenic factors. After confirming the presence of hematospermia, physicians should perform a clinical evaluation, including clinical history and physical examination with DRE. Several previous studies evaluated the pollen extract early pain relief in patients suffering from chronic prostatitis/chronic pelvic pain syndrome $(5,6)$.

In 2006, Elist in a double-blind randomized placebo controlled study, reported the superiority of pollen extract versus placebo in terms of pain score improvement and treatment of LUTS during six months of therapy (10). Additionally, in 2009, Wagenlehner et al. showed that pollen extract improved LUTS, pain and quality of life after 12 weeks of treatment in patients when compared with placebo (11). In our study, 2 weeks of treatment with DEPROX $500^{\circledR}$ provided significant results in terms of hematospermia disappearance.

This effect is possibly due to the association between the pollen extract and vitamins B6 and B12 that improve the antioxidant activity of pollen extract. In the study by Cai et al., DEPROX $500^{\circledR}$ resulted able to provide improved results in terms of early pain reduction in patients with non-inflammatory chronic prostatitis/chronic pelvic pain syndrome (6).

\section{Conclusions}

The administration of DEPROX $500^{\circledR}$ was able to achieve the complete resolution of hematospermia with a disappearance of this onset symptom in all patients within 15 days of treatment assumption, without side-effects.

Nevertheless, to completely resolve the bothering symptoms of xanthogranulomatous prostatitis surgery was mandatory in all patients. statitis: an unusual onset of a rare diagnosis. Can Urol Assoc J. 2013; 7:E820-E822.

9. Stillwell TJ, Engen DE, Farrow GM. The clinical spectrum of granulomatous prostatitis: a report of 200 cases. J Urol. 1987; 138:320-3.

10. Wagenlehner FM, Schneider H, Ludwig M, et al. A pollen extract (Cernilton) in patients with inflammatory chronic prostatitis-chronic pelvic pain syndrome: a multicentre, randomised, prospective, double-blind, placebo-controlled phase 3 study. Eur Urol. 2009; 56:544-551.

11. Elist J. Effects of pollen extract preparation Prostat/Poltit on lower urinary tract symptoms in patients with chronic nonbacterial prostatitis/chronic pelvic pain syndrome: a randomized, double blind, placebo-controlled study. Urology. 2006; 67:60-63.

\section{Correspondence}

Antonio Luigi Pastore, MD, PhD (Corresponding Author) - antopast@hotmail.com Yazan Al Salhi, MD

Andrea Fuschi, MD

Alessia Martoccia, MD

Gennaro Velotti, MD

Lorenzo Capone, MD

Antonio Carbone, MD - antonio.carbone@uniromal.it

Sapienza University of Rome, Faculty of Pharmacy and Medicine, Department of Medico-Surgical Scienes and Biotechonologies, Urology Unit, ICOT, Latina (Italy)

Natale Porta, MD

Vincenzo Petrozza, MD

Sapienza University of Rome, Faculty of Pharmacy and Medicine, Department of Medico-Surgical Sciences and Biotechnologies, Pathology Unit, Latina Italy

Giorgio Bozzini, MD

Department of Urology Mater Domini Humanitas Castellanza (Italy)

Ester Illiano, MD

Elisabetta Costantini, MD

Department of Urology and Andrology, University of Perugia, Terni (Italy) 